\title{
Erratum: Simulation of Tokamak SOL and Divertor Region Including Heat Flux Mitigation by Gas Puffing
}

\author{
[J. Korean Phys. Soc. 61, 387 (2012)] \\ Jin-Woo PARK, Yong-Su NA* and Sang Hee Hong \\ Department of Nuclear Engineering, Seoul National University, Seoul 151-742, Korea \\ Joon-Wook AHN \\ Oak Ridge National Laboratory, Fusion Energy Division, Oak Ridge, USA \\ Deok-Kyu KIM \\ Agency for Defense Development, Daejeon 305-152, Korea \\ Hyunsun HAN \\ National Fusion Research Institute, Daejeon 305-806, Korea \\ Seong Bo Shim and Hae June LEE \\ Department of Electrical Engineering, Pusan National University, Busan 609-735, Korea
}

DOI: $10.3938 / \mathrm{jkps} .61 .663$

One of the co-author's first name is corrected from 'Seong Bo Shim' to 'Seung Bo Shim'. 